\title{
Diamond Technology for High Current Density Patterned Cathodes
}

\author{
J.L. Shaw, J.E. Yater, J.E. Butler \\ Naval Research Lab \\ 4555 Overlook Ave., SW, Washington, D.C., USA 20375 \\ jon.shaw@nrl.navy.mil
}

\begin{abstract}
We consider the material quality requirements, potential performance, and fabrication processes needed to create a cold cathode from a freestanding diamond film, irradiated from the backside with an electron beam.
\end{abstract}

Keywords: cold cathode; diamond; negative electron affinity; freestanding film; electron beam.

\section{Introduction}

Electron sources that can be finely patterned as well as provide very high current density are needed for many vacuum electronic devices, especially topologies lacking cylindrical symmetry and/or requiring very small beam dimensions. Cold cathodes are preferred for such applications. A cold electron source can be made from a ptype semiconductor having negative electron affinity (NEA) when electrons are promoted to the conduction band, typically using either photons or electrons. The hydrogen-terminated diamond [111] or [100] surfaces have NEA, and are chemically robust and easily regenerated by exposure to atomic hydrogen. Electrons are preferred for electron-hole pair excitation since UV photons $(\lambda<225 \mathrm{~nm})$ would be required. E-beam illumination is most practical from the back of a freestanding film (fig. 1)[1,2]. The injected holes may be removed via conducting film on the primary beam side. A patterned metal coating on the emitting side can be used to define the emitting area and remove any electrons that are not emitted.

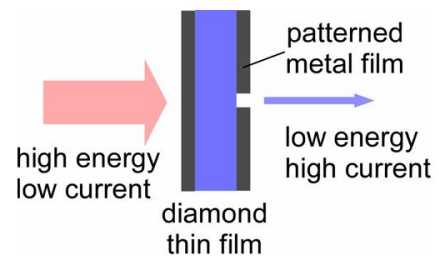

Figure 1.

We can define an emission efficiency $\eta=I_{E} / I_{G}$, where $I_{E}$ is the emission current and $I_{\mathbb{G}}$ is the generation current. To maximize $\eta$, the electrons and holes created by the primary beam must move to opposite surfaces without recombining. If the diamond is doped p-type, the electrons will drift randomly until they are emitted or recombine with holes. Because of random drift, $\eta$ will decline with film thickness and the density of scattering and recombination sites (point defects including dopant atoms and extended defects). In addition, the transit time through the film will be relatively long in films with high $\eta$.

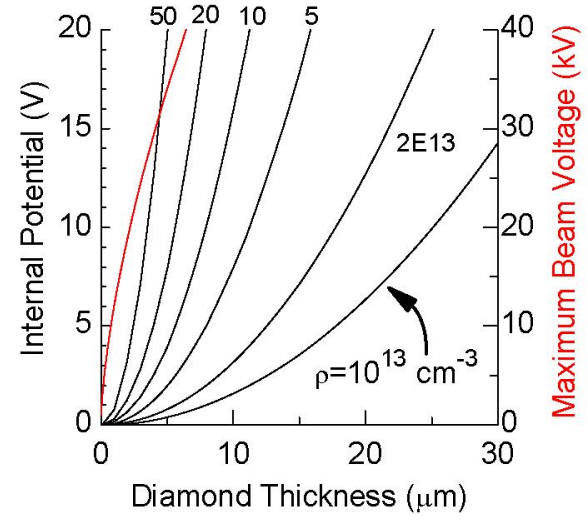

Figure 2.

Alternatively, the diamond might be intrinsic, with a small net positive charge (negative potentials will prevent emission). The resulting potential gradient inside the diamond will force the electrons to the NEA surface, reducing the transit time and increasing $\eta$. Diamond surfaces terminated with hydrogen exhibit some conductivity and are likely to screen most external fields, so the internal electric field must come from trapped positive charge rather than remote electrodes. Point defects in the upper part of the band gap, often related to nitrogen, typically become positively charged. The internal potentials that result from uniform charge densities $\rho$ in the range $1-50 \times 10^{13} \mathrm{~cm}^{-3}$ are plotted in figure 2 as a function of film thickness. Mechanical and fabrication constraints as well as the electron penetration depth (plotted on the red curve) set the minimum film thickness near 3-5 microns. The energy of the emitted electrons will range from the vacuum level at the surface to somewhat higher energies. The upper energy is determined by the scattering length, and will increase when there is a large electric field below the surface. The band bending and broadening effect is illustrated in figure 3 for the case $\rho=10^{14} \mathrm{~cm}^{-3}$. Thus both the absolute potential of the emitted beam and the energy distribution will be a function of $\rho$. The potential may also vary with time and position across the film surface if the fraction of charged point defects changes. To minimize these effects the charge concentration must be kept low, and/or the film must be kept thin. Thus $\rho$ must be below about $5 \times 10^{14} \mathrm{~cm}^{-3}$ to keep the surface potential below $15 \mathrm{~V}$ for $\mathrm{t}=4 \mu \mathrm{m}$, and relatively thick films $(\mathrm{t}=30 \mu \mathrm{m})$ may require $\rho<10^{13} \mathrm{~cm}^{-3}$, depending on the scattering length in the diamond and the required maximum energy distribution. 


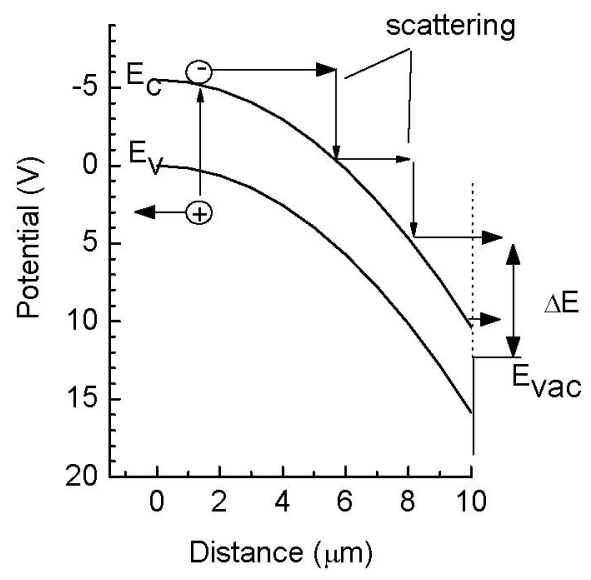

Figure 3.

Single crystal diamond grown using the high-pressure, high-temperature (HPHT) method (and most natural diamond) is unsuitable because it generally contains excessive nitrogen. Relatively pure, single crystal diamond can be grown on HPHT substrates using chemical vapor deposition. The nitrogen content of CVD films is limited by impurities in the feed gases; concentrations as low as $3 \times 10^{13} \mathrm{~cm}^{-3}$ have been demonstrated [3]. Other results indicate that high pressure annealing can improve the film quality, probably by altering the configuration of incorporated nitrogen [4]. NRL has also demonstrated a method of removing the CVD layers from the HPHT substrates to create freestanding films as thin as a few microns. Such films could be bonded to a suitable substrate (e.g. silicon) having micro machined slots to create a usable cathode.

Heating by limit the maximum emission current. Heating occurs due to the primary beam power plus transport loss, which is proportional to the total potential across the film, $\mathrm{V}_{\mathrm{S}}$. Ignoring losses in the front metal, the generation current $I_{g}$ relative to the primary beam power $P_{p}$ is $\mathrm{I}_{\mathrm{g}} / \mathrm{P}_{\mathrm{p}} \sim 1 /\left(3 \mathrm{E}_{\mathrm{g}}\right) \sim 60 \mathrm{~mA} /$ Watt. The heating power is thus approximately $\mathrm{P}_{\mathrm{S}}=\mathrm{P}_{\mathrm{P}}\left(1+0.06 \mathrm{~V}_{\mathrm{S}}\right)$.

A line emitter producing a sheet beam is illustrated in figure 4. This geometry is favorable for heat dissipation. Assuming the half-length $\mathrm{L}$ of the thin film is much greater than the film thickness $t$, and that the adjacent (thicker) material remains at room temperature, the temperature rise $\Delta \mathrm{T}$ can be estimated as $\Delta \mathrm{T}=\mathrm{P}_{\mathrm{S}}(\mathrm{L} / 2 \kappa \mathrm{t})$, where $\kappa$ is the thermal conductivity of diamond $(\sim 2000 \mathrm{~W} / \mathrm{m}-\mathrm{K})$. The maximum temperature must be less than the desorption

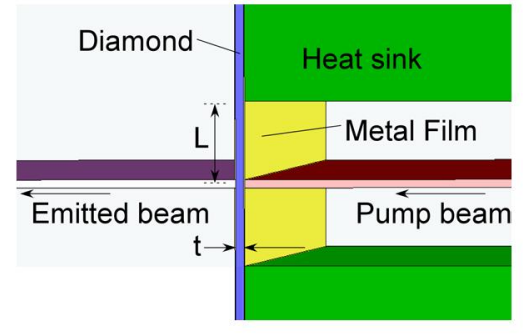

Figure 4.

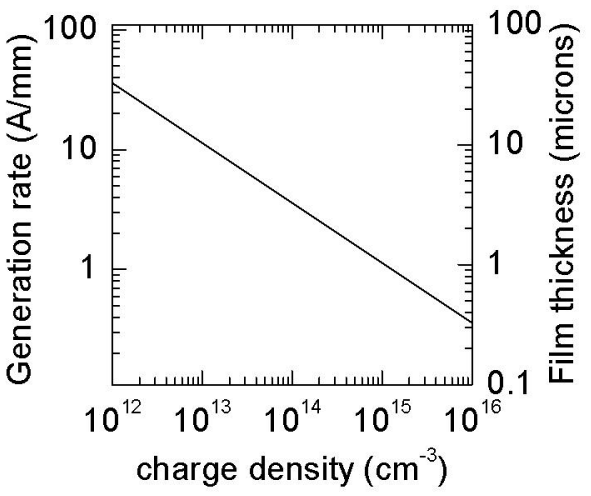

Figure 5.

temperature of hydrogen from diamond, $700-800 \mathrm{C}$. Assuming $\triangle \mathrm{T}<500 \mathrm{~K}$ and $\mathrm{L}=100 \mu \mathrm{m}$, the maximum power deposited in the specimen $\mathrm{P}_{\mathrm{S}}(\mathrm{W} / \mathrm{m})=\mathrm{t}(\mu \mathrm{m}) \times 2 \mathrm{E} 4$. In figure 5 we relate the generation rate (based on $P_{S}$ ) to the charge density by assuming $\mathrm{V}_{\mathrm{S}}=1.6 \mathrm{~V}$.

Lateral current transport across the diamond may also limit the emission current. The emission and transport properties of diamond are not presently understood well enough to make this calculation.

\section{Summary}

Production of outstanding emission current densities using e-beam pumped diamond appears possible, but will require diamond films with high crystal quality and low nitrogen content to avoid excessive charging, development of appropriate fabrication methods, and analysis of the emission current and energy distribution vs. primary beam power and diamond properties.

\section{References}

1. J. E. Yater, A. Shih, J. E. Butler, and P. E. Pehrsson, "Electron transmission studies of diamond films" Appl. Surf. Sci. vol. 191, pp. 52-60, 2002.

2. J. E. Yater, A. Shih, J. E. Butler, and P. E. Pehrsson, "Transmission of low-energy electrons in boron-doped nanocrystalline diamond films" J. Appl. Phys. Vol. 93, pp. 3082-3089, 2003.

3. J. E. Butler, M. W. Geis, K. E. Krohn, J. Lawless Jr, S. Deneault, T. M. Lyszczarz, D. Flechtner and R. Wright, "Exceptionally high voltage Schottky diamond diodes and low boron doping" Semiconductor Science and Technology Vol. 18, pp. S67-S71, 2003.

4. S. J. Charles, J. E. Butler, B. N. Feygelson, et. al. "Characterization of nitrogen doped chemical vapor deposited single crystal diamond before and after high pressure, high temperature annealing" Phys. Stat. Sol. A, Vol. 201, pp. 2473-2485, 2004. 